PSFC/JA-97-8

\title{
MODE CONVERSION TO ION-BERNSTEIN WAVES OF FAST ALFVÉN WAVES WITH POLOIDAL WAVENUMBERS IN SHEARED MAGNETIC FIELDS
}

\author{
S. D. Schultz, A. Bers, and A. K. Ram
}

April 1997

Plasma Science and Fusion Center Massachusetts Institute of Technology Cambridge, Massachusetts 02139 USA

This work was supported in part by DOE Grant No. DE-AC02-78ET51013 through TFTR and DOE Grant No. DE-FG02-91ER-54109. Reproduction, translation, publication, use and disposal, in whole or part, by or for the United States Government is permitted.

To be published in Proceedings of the 12th Topical Conference on Radio Frequency Power in Plasmas. 


\title{
MODE CONVERSION TO ION-BERNSTEIN WAVES \\ OF FAST ALFVÉN WAVES \\ WITH POLOIDAL WAVENUMBERS \\ IN SHEARED MAGNETIC FIELDS
}

S. D. Schultz, A. Bers, and A. K. Ram

\section{TABLE OF CONTENTS}

\begin{abstract}
. . . . . . . . . . . . . . . . . 1
Introduction . . . . . . . . . . . . . . . . . . . 1

Slab Geometry Model . . . . . . . . . . . . . . . . . . . . . . . 2

Dispersion Relation . . . . . . . . . . . . . . . . . . . . . . 2

Numerical Results . . . . . . . . . . . . . . . . . . . . . 3

Conclusions . . . . . . . . . . . . . . . . . . . . . . 4

Acknowledgements ... . . . . . . . . . . . . . . . . 4

References . . . . . . . . . . . . . . . . . . . 4
\end{abstract}




\title{
Mode Conversion to Ion-Bernstein Waves of Fast Alfvén Waves With Poloidal Wavenumbers in Sheared Magnetic Fields
}

\author{
S. D. Schultz, A. Bers, and A. K. Ram \\ Plasma Science and Fusion Center, \\ Massachusetts Institute of Technology, Cambridge, MA 02139
}

\begin{abstract}
Previous theoretical analysis [1] of mode conversion of fast Alfvén waves (FAW) to ion-Bernstein waves (IBW) neglected poloidal variations of the fields and magnetic shear. Full-wave codes show incident FAW fields focused into multiple poloidal modes near the ion-ion hybrid resonance (IHR)[2]. A new mode conversion model has been constructed including poloidal wavenumbers and poloidal magnetic field. The model consists of two coupled equations for the perpendicular components of the FAW electric field. These equations are numerically integrated to find the fraction of FAW power resonantly absorbed at the IHR. When the high-field-side right-hand cutoff is included, nonzero poloidal wavenumbers can change the triplet resonator phase and improve mode conversion at low $k_{\|}$.
\end{abstract}

\section{INTRODUCTION}

Recent experiments on TFTR [3] and Tore-Supra [2] have studied the use of mode-converted ion Bernstein waves (IBW) for electron heating and possible current drive [4]. The IBW's result from mode conversion of externally launched fast Alfvén waves (FAW) at the ion-ion hybrid layer, which can exist in a plasma with two or more ion species. IBW's have been proven to be efficent for electron heating, and are believed to result in synergistic current drive enhancement when used in conjunction with lower hybrid current drive [5].

An estimate for the mode conversion efficiency can be made using a cold plasma model and calculating the resonant FAW absorption which occurs at the ion-ion hybrid resonance [1]. This efficiency is found to be dependent primarily upon two parameters: the tunneling distance between the lefthand FAW cutoff and the ion-ion hybrid resonance, and the phase difference between the incident and reflected waves at the far end of the plasma, where the right-hand wave cutoff produces total reflection.

In this paper, we use the same resonant absorption model to calculate mode conversion efficiency, but include nonzero values of the poloidal wavenumber as well as a poloidal magnetic field. This gives a better approximation to the true wave spectrum which is launched by an antenna of finite 
length. In addition, the full wave code ALCYON [2] has shown that the FAW fields will have a strong poloidal variation due to focusing by the tokamak shape. It is also clear that magnetic field shear will cause a rotation of the FAW polarization which affects the FAW propagation equations.

\section{SLAB GEOMETRY MODEL}

The mode conversion region is approximately described by a set of Cartesian coordinates, with the $x$ direction chosen along the equatorial plane of the torus, and the $y$ and $z$ directions corresponding to the poloidal and toroidal directions respectively. The density and magnetic field of the plasma vary only in the $x$ direction. The magnetic field vector lies in the $y$ - $z$ plane, forming an angle $\alpha(x)$ with the $z$ axis. To calculate the dispersion relation for fast Alfvén waves, we rotate the coordinate frame into the direction of the magnetic field, so that at each point $x$ the coordinates must be rotated by $\alpha(x)$. The electric field of the FAW is assumed to have the form

$$
\vec{E}=\hat{E}(x) \exp \left(i k_{y} y+i k_{z} z-i \omega t\right)
$$

where $k_{y}$ and $k_{z}$ are treated as a constant in the vicinity of the mode conversion region. In the rotated coordinate system the wavenumbers become

$$
\begin{aligned}
k_{\|} & =k_{y} \sin \alpha+k_{z} \cos \alpha \\
k_{T} & =k_{y} \cos \alpha-k_{z} \sin \alpha .
\end{aligned}
$$

The dispersion relation of the fast Alfvén wave is calculated from cold plasma theory, using the approximation that for a low frequency wave, the electric field in the direction of the equilibrium magnetic field $\left(\hat{E}_{\|}\right)$will be shorted out.

\section{DISPERSION RELATION}

The dispersion relation for the FAW gives us a set of two coupled equations for the remaining wave components $\hat{E}_{x}$ and $\hat{E}_{T}$ :

$$
\begin{aligned}
\frac{d \hat{E}_{T}}{d \xi}= & -\frac{1}{n_{T}}\left[\left(D-\psi^{\prime} n_{\|}\right) \hat{E}_{T}+i\left(S-n_{T}^{2}-n_{\|}^{2}\right) \hat{E}_{x}\right] \\
\frac{d \hat{E}_{x}}{d \xi}= & -\frac{1}{\left(S-n_{\|}\right)}\left[i\left(D^{\prime}-n_{T} S+n_{T} n_{\|}^{2}\right) \hat{E}_{T}\right. \\
& \left.-\left(S^{\prime}-n_{T} D\right) \hat{E}_{x}+i\left(D-2 \psi^{\prime} n_{\|}\right) \frac{d \hat{E}_{T}}{d \xi}\right]
\end{aligned}
$$

In this notation, $\xi=(\omega x / c)$, and the primes all indicate differentiation with respect to $\xi$. $S$ and $D$ are the usual Stix tensor elements. 
(a)

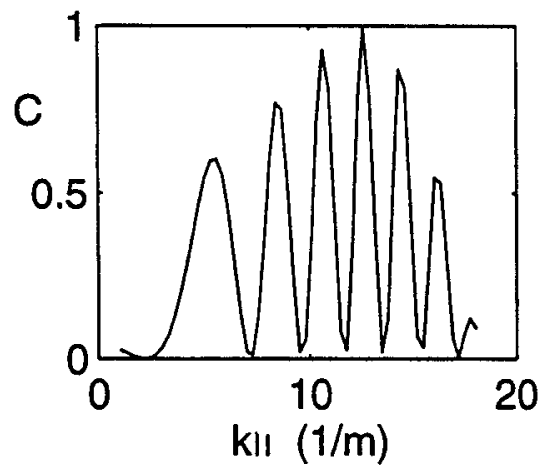

(b)

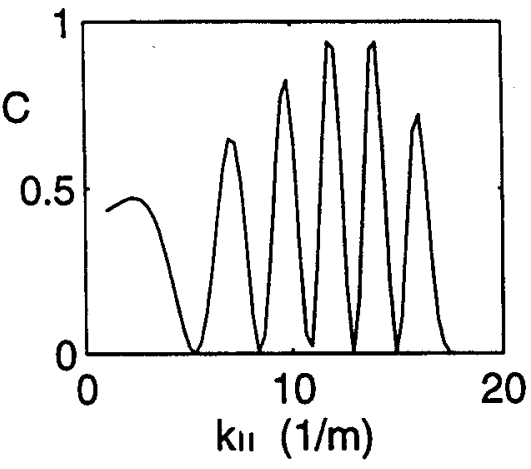

FIGURE 1. Fraction of FAW power dissipated $(C)$ as a function of $k_{\|}$for (a) $k_{T}=0$ and (b) $k_{T}=8 \mathrm{~m}^{-1}$, for a plasma with TFTR parameters.

Studying these equations, we observe that (3) is singular at the ion-ion hybrid layer where $S-n_{\|}^{2}=0$. Thus $\hat{E}_{x}$ becomes infinite and resonant absorption occurs. We also observe that if $n_{T}=0$, then (2) contains a division by zero, and the two equations are no longer coupled. In this case, we return to the previous mode-conversion theory [1], which makes use of a single second-order differential equation.

\section{NUMERICAL RESULTS}

The numerical integration of (2) and (3) is performed with boundary conditions appropriate to total wave reflection at the right hand FAW cutoff. The plasma parameters were chosen to fit the features of TFTR during modeconversion heating experiments [3]. By integrating to a point inside the plasma but near the antenna, we can compare the flux of power into and out of the mode conversion region, and find the fraction of power which was resonantly absorbed. Figure 1 shows the fraction of power mode converted for two cases, with $k_{T}=0$ and $k_{T}=8 \mathrm{~m}^{-1}$. As can be seen, the curves appear similar, but the phase of reflection at the right hand cutoff has been significantly changed by the finite poloidal wavenumber. We notice especially that at low values of $k_{\|}$(less than $3 \mathrm{~m}^{-1}$ ), the added poloidal features give mode-conversion fractions up to $50 \%$ where there was little mode conversion for $k_{T}=0$.

It is also possible to integrate the dispersion equations from the point of total reflection at the far end of the plasma, all the way back to the FAW antenna. At this point an antenna impedance can be calculated, and we can predict how well power will be coupled into the plasma, which acts like a resonator. In Figure 2 we show another set of numerical calculations based on this global resonator model, for plasma parameters found in Tore Supra mode-conversion experiments [2]. Figure 2 shows the spectral plasma impedance for modes with $k_{T}=0$ and $k_{T}=8 \mathrm{~m}^{-1}$. The peaks, which correspond to good power coupling to the plasma, are found at lower values 

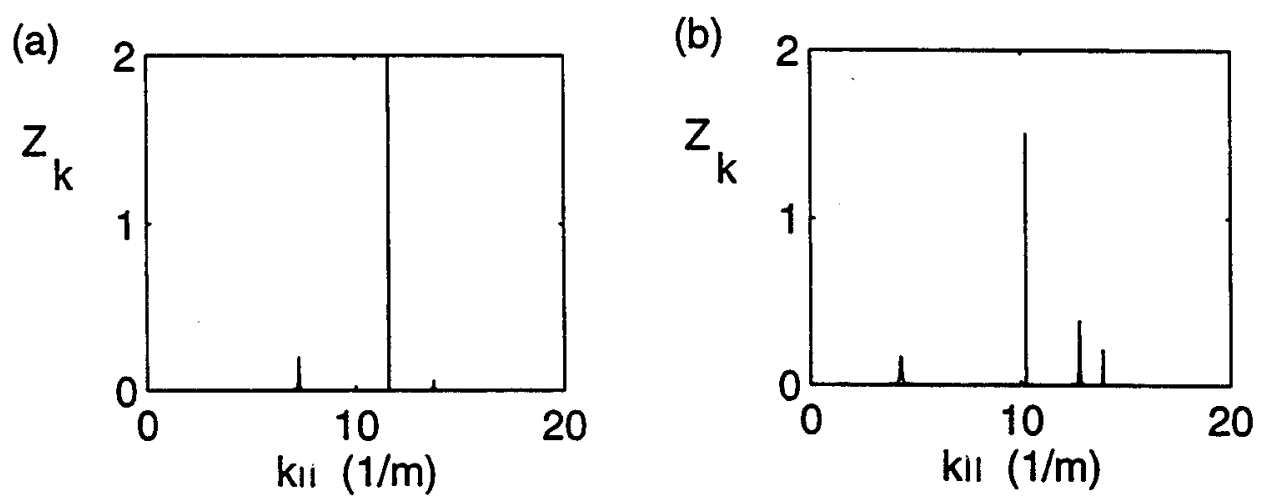

FIGURE 2. Spectral plasma impedance $\left(Z_{k}\right)$ as a function of $k_{\|}$for (a) $k_{T}=0$ and (b) $k_{T}=8 \mathrm{~m}^{-1}$, for a plasma with Tore Supra parameters.

of $k_{\|}$as we increase $k_{T}$. In the Tore-Supra experiments, which were analyzed using ALCYON, power deposition was observed over a broad range of $k_{\|}$ values, extending to very low $k_{\|}$. Our impedance calculations show that this cannot be predicted by calculations with $k_{T}=0$, but only by including a broad spectrum of poloidal modes.

\section{CONCLUSIONS}

A model for fast Alfvén wave propagation and mode conversion efficiency has been constructed which keeps the simplicity of a one-dimensional calculation but includes the important tokamak features of poloidal wavenumber and poloidal magnetic field. Using this model, it is shown that increasing $k_{T}$ can move the peaks of resonant absorption to lower values of $k_{\|}$. Therefore, correct modeling of the mode conversion efficiency at low $k_{\|}$requires particular attention to the spectrum of poloidal modes which are generated.

\section{ACKNOWLEDGEMENTS}

This work is supported by DoE Grant No. DE-AC02-78ET-51013 through TFTR and DoE Grant No. DE-FG02-91ER-54109.

\section{REFERENCES}

[1] Ram, A.K., Bers, A., Fuchs, V., and Schultz, S.D., Phys. Plasmas 3, 1976 (1996); Fuchs, V., Ram, A.K., Schultz, S.D., Bers, A., and Lashmore-Davies, C.N., Phys. Plasmas 2, 1637 (1995).

[2] Saoutic, B., et.al., Phys. Rev. Lett. 76, 1647 (1996).

[3] Majeski, R., et.al., Phys. Rev. Lett. 73, 2204 (1994).

[4] Becoulet, A., Plasma Phys. Control. Fusion 38, A1 (1996).

[5] Ram, A.K., Bers, A., Fuchs, V., and Harvey, R.W., in Proc. of the 10th Top. Conf. on Radio Freq. Power in Plasmas (Boston, MA 1993), Porkolab, M. and Hosea, J. Eds., Amer. Inst. of Phys. Conf. Proc. 289, New York (1994) 293-296. 